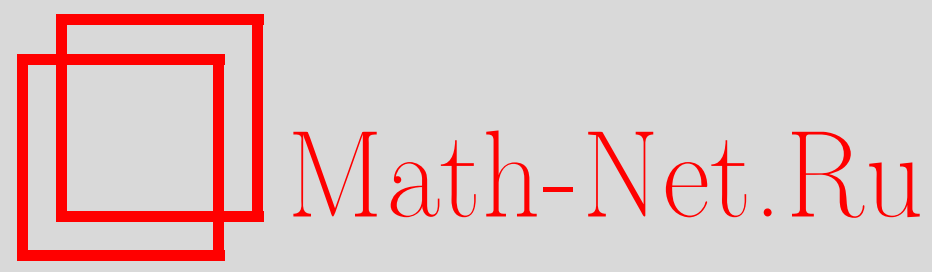

А. А. Туганбаев, Дистрибутивно порожденные кольца и дистрибутивные модули, Матем. заметки, 2000, том 68, выпуск 4, 568-578

DOI: https://doi.org/10.4213/mzm977

Использование Общероссийского математического портала Math-Net.Ru подразумевает, что вы прочитали и согласны с пользовательским соглашением http://www . mathnet.ru/rus/agreement

Параметры загрузки:

IP : 54.205 .225 .156

26 апреля 2023 г., 18:02:05

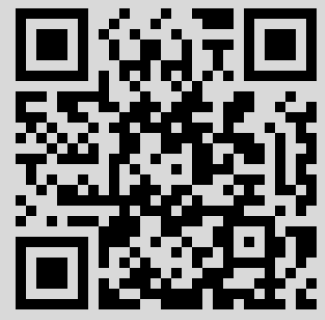




\section{ДИСТРИБУТИВНО ПОРОЖДЕННЫЕ КОЛЬЦА И ДИСТРИБУТИВНЫЕ МОДУЛИ}

\section{А. А. Туганбаев}

Модуль называется дистрибутивно порожденньм, если он порождается дистрибутивными подмодулями. Доказано, что кольцо эндоморфизмов конечно порожденного проективного правого модуля $M$ над дистрибутивно порожденньм справа кольцом является дистрибутивно порожденным справа кольцом. Если $M$ - модуль над кольцом $A$ и $A / J(A)$ - нормальное кольцо со свойством замены, то $M$ - дистрибутивньй модуль тогда и только тогда, когда $M$ - модуль Безу.

Библиограффия: 9 названий

1. Введение. В настоящей статье все кольца предполагаются ассоциативньми и (за исключением некоторых оговоренных случаев) с ненулевой единицей. Модуль $M$ назьвается дистрибутивным, если решетка всех его подмодулей дистрибутивна. Модуль назьвается дистрибутивно порожденным, если он порождается дистрибутивными подмодулями. Непосредственно проверяется, что каждая абелева группа является дистрибутивно порожденньм модулем над кольцом целых чисел. Прямая сумма дистрибутивных модулей назьвается полудистрибутивным модулем. Каждьй полудистрибутивньй модуль дистрибутивно порожден. Любая нециклическая и неквазициклическая неразложимая примарная абелева группа является дистрибутивно порожденньм неполудистрибутивным модулем над кольцом целых чисел. Если $F-$ поле и $A-$ 5 -мерная $F$-алгебра, порожденная всеми $(3 \times 3)$-матрицами вида

$$
\left(\begin{array}{ccc}
f_{11} & f_{12} & f_{13} \\
0 & f_{22} & 0 \\
0 & 0 & f_{33}
\end{array}\right), \quad f_{i j} \in F
$$

то можно проверить, что алгебра $A$ является полудистрибутивным справа полуцепным слева артиновым кольцом, не являющимся полуцепньм слева, и все (правые или левые) $A$-модули дистрибутивно порождены (см., например, [1, пример 1.22]). Полудистрибутивные модули и кольца изучаются в книге [1].

В [2] доказано, что все односторонние ниль-идеалы дистрибутивно порожденного справа кольца $A$ лежат в первичном радикале этого кольца. Первьй основной результат данной работы - теорема 1.

Работа поддержана Российским фондом фундаментальных исследований. 
ТЕОРемА 1. Пусть $M-$ конечно порожденный проективный правый модуль над дистрибутивно порожсденым справа кольцом. Тогда $\operatorname{End}(M)$ - дистрибутивно порохденное справа кольцо. Следовательно, все односторонние ниль-идеаль кольиа $\operatorname{End}(M)$ лежст в первичном радикале этого кольиа.

Кольцо $A$ называется строго регулярным, если для любого элемента $a \in A$ существует элемент $b \in A$ такой, что $a=a^{2} b$. Кольцо $A$ называется кольцом $с о$ свойством замены, если выполняются следующие два эквивалентных условия (см. [3]):

(i) для любого элемента $a \in A$ существует идемпотент $e \in a A$ такой, что $1-e \in$ $(1-a) A$

(ii) для любого элемента $a \in A$ существует идемпотент $e^{\prime} \in A a$ такой, что $1-e^{\prime} \in$ $A(1-a)$.

Если $M$ - модуль над кольцом $A$ и $A / J(A)$ - строго регулярное кольцо, то $M$ - дистрибутивный модуль $\Longleftrightarrow M-$ модуль Безу $[1,3.33]$. Вторым и третьим основньгми результатами данной работы являются теоремы 2 и 3.

ТЕОрема 2. Пусть $M-$ модуль над кольиом $A$ и $A / J(A)-$ нормальное кольио со

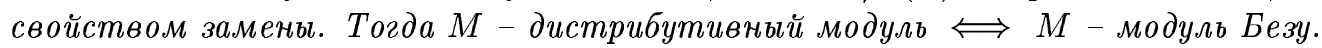

В связи с теоремой 2 заметим, что каждое строго регулярное кольцо является нормальным полупримитивным кольцом со свойством замены. Если $A$ - коммутативное кольцо и все простые идеалы кольца $A$ максимальны, то $A$ - нормальное кольцо со свойством замены. Если $A$ - прямое произведение бесконечного числа полей, то можно проверить, что кольцо формальных степенных рядов $A[[x]]$ является коммутативньм дистрибутивным кольцом со свойством замены, содержащим бесконечное множество ортогональных идемпотентов. Кроме того, существует коммутативное полупримитивное кольцо $A$ со свойством замены, которое не является строго регулярным кольцом. Пусть $\mathbb{Q}-$ поле рациональных чисел, $S$ - кольцо всех рациональных чисел с нечетными знаменателями, $R$ - прямое произведение счетного множества экземпляров поля $\mathbb{Q}, A$ - подкольцо кольца $R$, образованноевсеми последовательностями вида $\left(q_{1}, q_{2}, \ldots, q_{n}, s, s, s, \ldots\right)$, где $n \geqslant 1, q_{i} \in \mathbb{Q}, s \in S$. Тогда $A$ - требуемое кольцо.

Теорема 3. Для кольиа А со свойством замены равносильны следующие условия:

(1) $A$ - дистрибутивное справа кольио;

(2) $A$ - нормальное правое кольио Безу;

(3) $A$ - дистрибутивное слева кольио;

(4) $A$ - нормальное левое кольцо Безу;

(5) для кажсдого максимального правого или левого идеала $M$ кольца $A$ существует двустороннее кольио частных ${ }_{M} A_{M}$, которое является иепным справа кольцом, причем канонический кольчевой гомоморфизм $f: A \rightarrow{ }_{M} A_{M}$ сюргективен.

В связи с теоремой 3 заметим, что первая алгебра Вейля над полем нулевой характеристики является нормальным правым и левьм кольцом Безу, которое не является дистрибутивным справа или слева кольцом. Пусть $\mathbb{Z}$ - кольцо целых чисел, $A$ - подкольцо поля комплексных чисел, образованное числами $a+b \sqrt{5} i$, где $a, b \in \mathbb{Z}, i^{2}=-1$. Тогда $A$-коммутативная дистрибутивная область, не являющаяся кольцом Безу. Кроме того, 
для любого максимального идеала $M$ области $A$ канонический кольцевой гомоморфизм из кольца $A$ в кольцо частных $A_{M}$ не является сюръективным.

В работе доказаны и другие результаты о дистрибутивно порожденных и дистрибутивных модулях. В частности, доказано, что кольцо, эквивалентное в смысле Мориты дистрибутивно порожденному справа кольцу, является дистрибутивно порожденным справа кольцом (следствие 1(1) ). Рассматриваются дистрибутивно порожденные справа $\pi$-регулярные кольца и дистрибутивные модули над кольцами без единицы (предложения 6 и 7$)$.

2. Определения и обозначения. Через $J(M)$ и $\operatorname{End}(M)$ обозначаются соответственно радикал Джекобсона и кольцо эндоморфизмов модуля $M$. Модуль $M$ называется иепным м, если любые два его подмодуля сравнимы по включению. Прямая сумма цепньх модулей назьвается полуиепным модулем. Модуль $M$ назьвается модулем Безу, если каждьй конечно порожденньй подмодуль модуля $M$ является циклическим модулем. Кольцо называется нормальным, если все его идемпотенты центральны. Подмножество $T$ кольца $A$ назьвается мультипликативным, если $1 \in T, 0 \notin T$ и произведение любых элементов множества $T$ принадлежит $T$. Пусть $T$ - мультипликативное подмножество кольца $A$ такое, что существуют кольцо ${ }_{T} A_{T}$ и кольцевой гомоморфизм $f: A \rightarrow{ }_{T} A_{T}$ такие, что $f(T) \subseteq U\left({ }_{T} A_{T}\right),{ }_{T} A_{T}=\left\{f(a) f(t)^{-1} \mid a \in A, t \in T\right\}=$ $\left\{f(t)^{-1} f(a) \mid a \in A, t \in T\right\}$ и $\operatorname{Ker}(f)=K(T)$. В этом случае кольцо ${ }_{T} A_{T}$ назьвается двусторонним кольцом частны $x$ кольца $A$ относительно $T$, а гомоморфизм $f$ назьвается каноническим гомоморфизмом для ${ }_{T} A_{T}$. В том случае, когда $T=A \backslash M$, будем писать ${ }_{M} A_{M}$ вместо ${ }_{T} A_{T}$.

Модуль $M$ назьвается квазиинвариантным, если все его максимальные подмодули вполне инвариантны в $M$. Непосредственно проверяется, что $A-$ квазиинвариантное справа кольцо $\Longleftrightarrow$ все правые идеалы кольца $A$ являются идеалами. Кольцо без ненулевых нильпотентных элементов назьвается редуцированным кольцом. Кольцо $A$ назьвается иорновским, если $J(A)$ - ниль-идеал и каждый правьй идеал кольца $A$, не лежащий в $J(A)$, содержит ненулевой идемпотент. Кольцо $A$ называется $\pi$-регулярнылм, если для любого элемента $a \in A$ существует элемент $b \in A$ такой, что $a^{n}=a^{n} b a^{n}$ для некоторого натурального числа $n$. Непосредственно проверяется, что каждое $\pi$-регу-

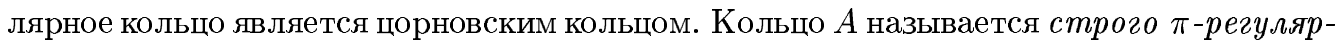
ны.м, если выполнены следуюшие два эквивалентных условия (см. [4]):

(i) для любого элемента $a \in A$ существует натуральное число $n$ такое, что $a^{n} A=$ $a^{n+1} A$

(ii) для любого элемента $a \in A$ существует натуральное число $m$ такое, что $A a^{m}=$ $A a^{m+1}$.

Кольцо $A$ называется кольцом индекса не более $n$, если существует натуральное число $n$ такое, что $a^{n}=0$ для любого нильпотентного элемента $a \in A$. Кольцо $A$ назьвается полусовершенным, если $A / J(A)$ - артиново кольцо и все идемпотенты кольца $A / J(A)$ поднимаются до идемпотентов кольца $A$. Подмножество $T$ кольца $A$ называется перестановочным справа (в $A$ ), если для любых элементов $a \in A$ и $t \in T$ существуют элементы $b \in A$ и $u \in T$ такие, что $a u=t b$. Подмножество $T$ кольца $A$ назьвается реверсивным справа, если для любых элементов $a \in A$ и $t \in T$ таких, что $t a=0$, существует элемент $u \in T$ такой, что $a u=0$.

Модуль назьвается равномерным, если любые два его ненулевых подмодуля имеют ненулевое пересечение. Модуль назьвается конечномерным, если он не содержит 
бесконечных прямых сумм ненулевых подмодулей. Подмодуль $N$ модуля $M$ называется существенным (в $M$ ), если $N$ имеет ненулевое пересечение с любым ненулевьп подмодулем модуля $M$. В этом случае мы будем говорить, что $M$ - существенное расширение модуля $N$. Правьй модуль $M$ над кольцом $A$ назьвается несингулярным, если правый аннулятор любого ненулевого элемента $m \in M$ не является существенным правым идеалом кольца $A$. Модуль $M$ назьвается нильпотентно инвариантным, если каждьй его нильпотентный эндоморфизм отображает все подмодули модуля $M$ в себя. Циклический модуль $M$ назьвается локальным, если $M / J(M)$ - простой модуль.

\section{3. Доказательство основных результатов.}

ЛЕмма 1. (1) Если модуль является суммой дистрибутивно порожсденных подмодулей, то этот модуль дистрибутивно порохсден.

(2) Если модуль $M$ - сумма дистрибутивных подмодулей $M_{i}(i \in I)$, то $M$ гомоморфный образ прямой суммы дистрибутивных модулей $M_{i}$.

(3) Каждый гомоморфный образ дистрибутивно порожденного модуля является дистрибутивно порохсденным модулем.

(4) Каждый правый модуль над дистрибутивно порохденным справа кольиом А дистрибутивно порохсен.

(5) Если $M$ - конечно порохсенный дистрибутивно порожденный модуль, то существуют дистрибутивные подмодули $M_{1}, \ldots, M_{n}$ модуля $M$ такие, что $M=$ $\sum_{i=1}^{n} M_{i}$ и $M-$ гомоморфный образ модуля $\bigoplus_{i=1}^{n} M_{i}$.

(6) Каждый конечно порохденный правый модуль над дистрибутивно порожденным справа кольцом является гомоморфным образом конечной прямой суммы дистрибутивных правых идеалов этого кольиа.

(7) Кажсдий локальный дистрибутивно порожденный модуль $М$ дистрибутивен.

ДокАЗАТЕЛЬСтво. (1) и (2) проверяются непосредственно.

(3) Пусть $h: M \rightarrow N$ - модульньй эпиморфизм и $M=\sum_{i \in I} M_{i}$, где все $M_{i}$ - дистрибутивные модули. Тогда все модули $h\left(M_{i}\right)$ являются дистрибутивными и $h(M)=$ $\sum_{i \in I} h\left(M_{i}\right)$.

(4) Согласно (1) каждый свободньй правьй $A$-модуль является дистрибутивно порожденным. Поэтому (4) следует из (3) и того, что каждьй модуль - гомоморфный образ свободного модуля.

(5) Пусть $M=\sum_{i \in I} M_{i}$, где все $M_{i}$ - дистрибутивные модули. Так как $M-$ конечно порожденньй модуль, то существует конечное подмножество $J$ в $I$ такое, что $M=$ $\sum_{i \in J} M_{i}$. Можно считать, что $J=\{1, \ldots, n\}$. Теперь (5) следует из (2).

(6) следует из (4) и (5).

(7) Согласно (5) $M=\sum_{i=1}^{n} M_{i}$, где все $M_{i}$ - дистрибутивные модули. Так как $M-$ локальный модуль, то $M$ совпадает с одним из дистрибутивных модулей $M_{i}$.

Лемма 2. Пусть е-ненулевой идемпотент кольиа $А$. Тогда имеют место следующие утверждения.

(1) Если $N$ - первичный радикал кольца $A$, то еNe-первичный радикал кольu, а eAe.

(2) Если первичный радикал $P$ кольиа $A$ содержит все правые ниль-идеалы кольиа $A$, то первичный радикал кольца еАе содержит все правые ниль-идеалы кольu, $а$ eAe. 
(3) Если $M$ - дистрибутивный правый $A$-модуль, то Ме - дистрибутивный правый еАе-модуль.

(4) Если $M$ - дистрибутивный правый идеал кольиа $A$, то еМе - дистрибутивный правый идеал кольиа еАе.

ДокАзАТЕЛьство. (1) Утверждение доказано в [5].

(2) Согласно (1) ePe- первичный радикал кольца $e A e$. Пусть $X=e X e=e X e A e-$ правый ниль-идеал кольца $e A e, a$ - элемент кольца $A$ и $x=e x e \in X$. Так как элемент ехеае принадлежит правому ниль-идеалу $X$ кольца $e A e$, то существует натуральное число $n$ такое, что $(\text { exeae })^{n}=0$. Тогда $(x a)^{n+2}=(\text { exea })^{n+2}=$ exea $(\text { exea })^{n}$ exea $=$ exea $(\text { exeae })^{n}$ exea $=0$. Поэтому $x A$-правый ниль-идеал кольца $A$. По предположению $x A \subseteq N$. Поэтому $x \in e P$ и $X \subseteq e P e$.

(3) доказано в $[1,1.48(3)]$.

(4) Согласно (3) $M e$ - дистрибутивный правьй $e A e$-модуль. Так как $e M e$ - гомоморфный образ дистрибутивного $e A e$-модуля $M e$, то $e M e-$ дистрибутивньй $e A e-$ модуль.

Лемма 3. Пусть $M-$ прямое слагаемое модуля $\bigoplus_{i=1}^{n} M_{i}$.

(1) Если все модули $M_{1}, \ldots, M_{n}$ нильпотентно инвариантны, то первичный радикал кольиа $\operatorname{End}(M)$ содержит все правые и все левье ниль-идеаль кольца $\operatorname{End}(M)$.

(2) Если все модули $M_{1}, \ldots, M_{n}$ дистрибутивны, то первичный радикал кольца $\operatorname{End}(M)$ содерэит все правые и все левые ниль-идеаль кольиа $\operatorname{End}(M)$.

ДоказАтельство. (1) Пусть $X \equiv \bigoplus_{i=1}^{n} M_{i}, R \equiv \operatorname{End}(X)$ и $P$-первичный радикал кольца $R$. Так как $X$ - конечная прямая сумма нильпотентно инвариантных модулей, то $P$ содержит все правые или левые ниль-идеалы кольца $R[1,8.28(1)]$. Существует идемпотент $e \in R$ такой, что $M=e(X)$ и $\operatorname{End}(M)=e R e$. По лемме $2(2)$ первичньй радикал кольца $e R e$ содержит все правые или левые ниль-идеалы кольца $e R e$.

(2) следует из (1) и из того, что каждый дистрибутивньй модуль является нильпотентно инвариантным по $[1,8.24(5)]$.

ПРЕДЛОЖЕНИЕ 1. Пусть проективный модуль $M$ порождается подмодулями $M_{1}, \ldots, M_{n}$ и $P$ - первичный радикал кольиа $\operatorname{End}(M)$. Тогда имеют место следующие утверэдения.

(1) Модуль $M$ изоморфен прямому слагаемому модуля $\bigoplus_{i=1}^{n} M_{i}$.

(2) Если все модули $M_{1}, \ldots, M_{n}$ нильпотентно инвариантны, то $P$ содержит все правые и все левье ниль-идеалы кольца $\operatorname{End}(M)$.

(3) Если все модули $M_{1}, \ldots, M_{n}$ дистрибутивны, то $P$ содержит все правые и все левые ниль-идеаль кольиа $\operatorname{End}(M)$.

ДокАзАТЕЛьСТво. (1) следует из существования эпиморфизма модуля $\bigoplus_{i=1}^{n} M_{i}$ на проективньй модуль $M$.

(2) и (3) следуют из (1) и леммы 3.

ПРЕДЛОЖЕНИЕ 2. Пусть $M$ - конечно порожденный проективный дистрибутивно порожденный модуль. Тогда все односторонние ниль-идеаль кольца $\operatorname{End}(M)$ лехсат в первичном радикале этого кольца.

ДокАЗАТЕЛЬСТВо. Предложение 2 следует из леммы 1(5) и предложения 1(3). 
ПРЕДЛОЖЕНИЕ 3. Пусть $A$ - дистрибутивно порожденное справа кольцо. Тогда имеют место следующие утверждения.

(1) Каждый гомоморфный образ кольиа $A$ является дистрибутивно порожденным справа кольцом.

(2) Кольчо еАе является дистрибутивно порожденным справа кольцом для любого идемпотента $е \in A$.

(3) Для любого натурального числа $s$ кольцо матрии $A_{s}$ является дистрибутивно порохсденным справа кольцом.

ДокАЗАТЕЛЬСТво. Пусть $A=\sum_{i=1}^{n} M_{i}$, где все $M_{i}$ - дистрибутивные правые идеалы кольца $A$.

(1) проверяется непосредственно.

(2) По лемме 2(4) все $e M_{i} e$ - дистрибутивные правые идеалы кольца $e A e$. Кроме того, $e A e=e\left(\sum_{i=1}^{n} M_{i}\right) e=\sum_{i=1}^{n} e M_{i} e$. Поэтому $e A e-$ дистрибутивно порожденное справа кольцо.

(3) Пусть $R \equiv A_{s}$ и $R_{k}$ - правьй идеал кольца $R$, образованньй всеми матрицами $\left(a_{i j}\right)$, у которых $a_{i j}=0$ при $i \neq k(k=1, \ldots, s)$. Так как $R_{R}$ - прямая сумма изоморфных правьх идеалов $R_{k}(k=1, \ldots, s)$, то достаточно доказать, что $R_{1}$ - дистрибутивно порожденньй правый $R$-модуль. Так как $A=\sum_{i=1}^{n} M_{i}$, то правьй $R$-модуль $R_{1}$ является суммой своих подмодулей $X_{1}, \ldots, X_{n}$, где $X_{i}-$ множество всех матриц из $R_{1}$ таких, что $a_{1 j} \in M_{i}$ для всех $j$. Так как все $M_{i}$ - дистрибутивные правые $A$-модули, то все $X_{i}$ - дистрибутивные правые $R$-модули. Поэтому $R_{1}$ - дистрибутивно порожденньй правый $R$-модуль.

ДОКАЗАТЕЛЬСТВО ТЕОРЕМЫ 1 . Пусть $n$ - натуральное число, $M-n$-порожденный проективньй правьй модуль над дистрибутивно порожденным справа кольцом $A$ и $P$ первичный радикал кольца $\operatorname{End}(M)$. По лемме $1(4) \quad M$ дистрибутивно порожден. По предложению 2 радикал $P$ содержит все правые и все левые ниль-идеалы кольца $\operatorname{End}(M)$. Так как $M-n$-порожденный проективный $A$-модуль, то модуль $M$ изоморфен прямому слагаемому прямой суммы $n$ изоморфных копий модуля $A_{A}$. Поэтому существует идемпотент $e$ кольца матриц $A_{n}$ такой, что кольцо $\operatorname{End}(M)$ изоморфно кольцу $e A_{n} e$. По предложению $3 \operatorname{End}(M)$ - дистрибутивно порожденное справа кольцо.

СледСтВИЕ 1. Пусть $A$ - дистрибутивно порожденное справа кольцо. Тогда имеют место следующие утверждения.

(1) Если $B$ - кольчо такое, что категория правых В-модулей әквивалентна категории правых $A$-модулей, то $B$ - дистрибутивно порожсднное справа кольио.

(2) Если $J(A)$ - ниль-идеал кольиа $A$, то $J(A)$ совпадает с первичным радикалом $P(A)$ кольиа $A$.

(3) Если $A$ - иорновское кольцо, то $J(A)$ совпадает с первичным радикалом $P(A)$ кольиа $A$.

(4) Если $A$ - полупервичное кольцо и $J(A)$ - ниль-идеал, то $A$ - полупримитивное кольио.

(5) Если $A$ - чорновское полупервичное кольцо, то $A$ - полупримитивное коль$u, 0$.

ДокАЗАТЕЛЬСТВо. (1) вытекает из теоремы 1 и того, что кольцо $B$ изоморфно кольцу эндоморфизмов конечно порожденного проективного правого $A$-модуля $[6,12.10]$.

(2) По теореме $1 J(A) \subseteq P(A)$. Обратное включение верно для любого кольца. 
(3) следует из того, что радикал Джекобсона цорновского кольца является ниль-идеалом.

(4) и (5) следуют из (2) и (3).

Лемма 4. (1) Все регулярные кольиа и все фактор-кольиа колеи, со свойством замены являются кольцами со свойством замены.

(2) $A$ - кольцо со свойством замены без нетривиальных идемпотентов $\Longleftrightarrow$ $A$ - локальное кольцо.

(3) Кажсдое нормальное кольцо со свойством замены $А$ является квазиинвариантным справа и слева кольцом.

(4) Пусть $A$ - кольцо такое, что $A / J(A)$ - нормальное кольио со свойством замены. Тогда $A$ - квазиинвариантное справа и слева кольцо и $A / J(A)$ - редуцированное кольио. Следовательно, $J(A)$ содерэит все нильпотентные әлементы кольиа $A$.

ДокАЗАТЕЛЬСтво. (1) и (2) проверяются непосредственно.

(3) Предположим, что максимальньй правьй идеал $M$ кольца $A$ не является идеалом. Тогда $A=M+A M$. Существуют элементы $m_{1}, m_{2} \in M$ и $a \in A$ такие, что $m_{1}+a m_{2}=1$. Так как $A-$ нормальное кольцо со свойством замены, то существует центральньй идемпотент $e \in m_{1} A \subseteq M$ такой, что $1-e \in\left(1-m_{1}\right) A=a m_{2} A$. Поэтому существует элемент $m_{3} \in m_{2} A \subseteq M$ такой, что $1-e=a m_{3}$. Тогда $m_{3} a$ - центральньй идемпотент. Поэтому $1-e=a\left(m_{3} a\right) m_{3}=\left(m_{3} a\right) a m_{3} \in M$. Тогда $1=e+(1-e) \in M$; получено противоречие.

(4) Согласно (3) $A / J(A)$ - квазиинвариантное кольцо. Тогда $A / J(A)$ - подпрямое произведение тел, откуда $A / J(A)$ - редуцированное кольцо. Так как $A / J(A)$ квазиинвариантно справа, то и само кольцо $A$ квазиинвариантно справа.

Лемма $5[1,1.17,3.47,3.26,8.25(10)]$. (1) Правый модуль $M$ над кольиом $А$ дистрибутивен $\Longleftrightarrow$ для любых двух әлементов $m, n$ модуля $M$ существуют әлементы $a, b, c, d$ кольиа $A$ такие, что $1=a+b$, ma $=n c$ u $n b=m d$.

(2) Если $M$ - дистрибутивный модуль, то $M$ квазиинвариантен $и \operatorname{End}(M)$ нормальное кольио, у которого все односторонние ниль-идеалы лежат в первичном радикале.

(3) Если $A$ - квазиинвариантное справа кольцо, то каждый правый $A$-модуль Безу является дистрибутивным модулем.

ПРЕДЛОЖЕНИЕ 4. Пусть $A$ - нормальное кольио со свойством замены $и M-$

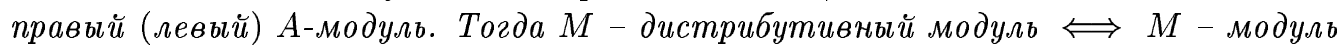
Безу.

ДокАЗАТЕЛЬСтво. Необходимость. Пусть $m, n \in M_{A}$. По лемме 5 сушествует элемент $a \in A$ такой, что $m a A+n(1-a) A \subseteq m A \cap n A$. Так как $A$ - нормальное кольцо со свойством замены, то существует центральньй идемпотент $e \in A$ такой, что $e \in a A$ и $1-e \in(1-a) A$. Тогда $m e A+n(1-e) A \subseteq m A \cap n A$. Поэтому me $\in$ ne $A$, $n(1-e) \in m(1-e) A$. Поэтому $N=N e \oplus N(1-e)=m e A+n e A+m(1-e) A+n(1-e) A=$ $(n e+m(1-e)) A$.

Достаточность. По лемме $4(3) A$ - квазиинвариантное кольцо. По лемме $5(3)$ модуль Безу $M_{A}$ дистрибутивен. 
ПРЕДЛОЖЕНИЕ 5. Пусть $A$ - нормальное кольцо со свойством замены $и M-$ максимальный правый или левый идеал кольца А. Тогда существует двустороннее кольцо частных ${ }_{M} A_{M}$, которое является локальным кольцом, и канонический кольиевой гомоморфизм $f: A \rightarrow{ }_{M} A_{M}$ сюрвективен.

ДокАЗАТЕЛЬСТво. Пусть $M$ - максимальный правьй идеал кольца $A$ и $T \equiv A \backslash M$. По лемме $4(3) M$ - идеал. Тогда $A / M$ - тело, откуда $T$ - мультипликативное множество. Докажем следующие утверждения:

(1) для любого $t \in T$ существуют элементы $u \in T$ и $m_{1}, m_{2} \in M$ такие, что $t u=1-m_{1} \in T$ и $u t=1-m_{2} \in T$

(2) $T$ - перестановочное справа множество;

(3) $T$ - реверсивное справа множество.

(1) следует из того, что $A / M$ - тело.

(2) Пусть $a \in A$ и $t \in T$. Согласно (1) существуют элементы $u \in T$ и $m \in M$ такие, что $t u=1-m \in T$. Так как $A$ - нормальное кольцо со свойством замены, существует центральньй идемпотент $e \in m A \subseteq M$ такой, что $1-e \in(1-m) A$. Поэтому

$$
a(1-e)=(1-e) a \in(1-m) A=t u A \subseteq t A
$$

Кроме того, $1-e \in T$, поскольку $e \in M$. Поэтому множество $T$ перестановочно справа.

(3) Пусть $a \in A, t \in T$ и $t a=0$. Согласно (1) существуют элементы $u \in T$ и $m \in M$ такие, что $u t=1-m \in T$. Тогда $(1-m) a=0$. Так как $A$ - нормальное кольцо со свойством замены, то существует центральньй идемпотент $e \in A m \subseteq M$ такой, что $1-e \in A(1-m)$. Поэтому $a(1-e)=(1-e) a \in A(1-m) a=0$. Кроме того, $1-e \in T$, поскольку $e \in M$. Поэтому множество $T$ реверсивно справа.

Из утверждений (1)-(3) следует, что $T$ - перестановочное справа и слева реверсивное справа и слева мультипликативное множество. В силу $[1,5.25,5.20(14)]$ сушествует двустороннее кольцо частных ${ }_{M} A_{M}$, которое является локальным кольцом, и $J\left(A_{M}\right)=$ $f(M) A_{M}$, где $f: A \rightarrow A_{M}$ - канонический кольцевой гомоморфизм. Тогда $f(A)$-кольцо со свойством замены. Кроме того, $f(A)$ не имеет нетривиальных идемпотентов, поскольку $f(A)$ - подкольцо локального кольца $A_{M}$. Поэтому $f(A)$ - локальное кольцо. Кроме того, все элементы из $f(M)$ необратимы в кольце $f(A)$, поскольку $J\left(A_{M}\right)=f(M) A_{M}$. Поэтому $f(M)=J(f(A))$. Тогда все элементы множества $f(T)$ обратимы в кольце $f(A)$, откуда $A_{M}=f(A)$ и $f: A \rightarrow A_{M}$ - сюръективньй гомоморфизм.

ДоКАЗАТЕЛЬСТво ТЕОРЕмЫ 2. Необходимость. Пусть $m, n \in M, N \equiv m A+n A$, $J \equiv J(A)$ и $h: N \rightarrow N / N J$ - естественный эпиморфизм. Достаточно доказать, что $N-$ циклический модуль. Так как $N J$ - малый подмодуль модуля $N_{A}$, достаточно доказать, что дистрибутивньй правьй $A / J$-модуль $h(N)$ является циклическим. Поэтому можно считать, что $J=0$ и $A$ - нормальное кольцо со свойством замены. В этом случае утверждение следует из предложения 4.

Достаточность. По лемме $4(3) A / J(A)$ - квазиинвариантное кольцо. Поэтому $A-$ квазиинвариантное кольцо. По лемме 5(3) модуль Безу $M_{A}$ является дистрибутивным.

ДоКАЗАТЕЛЬСтво тЕоРЕмы 3. Так как условие (5) теоремы лево-право симмметрично, то достаточно доказать эквивалентность условий (1), (2) и (5). (1) дует из предложения 4(1) и из того, что все дистрибутивные справа кольца являются нормальными кольцами по лемме $5(2) .(5) \Longrightarrow(1)$ является частным случаем $[1,7.8]$. 
$(1) \Longrightarrow(5)$ По предложению 5 сушествует двустороннее кольцо частных ${ }_{M} A_{M}$, которое является локальным кольцом, причем канонический кольцевой гомоморфизм $f$ : $A \rightarrow{ }_{M} A_{M}$ сюръективен. Согласно $[1,7.8]{ }_{M} A_{M}$ - цепное справа кольцо.

Лемма 6. Пусть $A$ - первичное кольцо. Тогда выполнены следующие утверждения.

(1) Каждыцй дистрибутивный правый идеал кольиа А является равномерным правым идеалом.

(2) Если $n$ - натуральное число, то каждый правый идеал кольиа А, являющийся суммой $n$ дистрибутивных правых идеалов, является существенным расширением прямой суммы $п$ равномерных правых идеалов.

ДокАЗАТЕЛЬСТво. (1) доказано в $[1,1.50(2)]$.

(2) Будем использовать индукцию по числу $n$. При $n=1$ утверждение следует из (1). Допустим, что утверждение верно для числа $n-1$ и $B$-правьй идеал кольца $A$ такой, что $B=\sum_{i=1}^{n} B_{i}$, где все $B_{i}$ - дистрибутивные правые идеалы. Положим $D \equiv \sum_{i=1}^{n-1} B_{i}$. Тогда $B / D$ - дистрибутивньй $A$-модуль, поскольку $B / D \cong B_{n} /\left(B_{n} \cap D\right)$. По предположению индукции $D$ - существенное расширение правого идеала $D^{\prime}$ такого, что $D^{\prime}=\bigoplus_{i=1}^{n-1} D_{i}$, где $k \leqslant n$ и все $D_{i}$ - равномерные правые идеалы. Существует правьй идеал $D_{n}$ кольца $A$ такой, что $B$ - сушественное расширение правого идеала $D+D_{n}$ и $D_{n} \cap D=0$. Тогда $B$ - существенное расширение правого идеала $D^{\prime}=\bigoplus_{i=1}^{n} D_{i}$. Так как $D_{n} \cap D=0$, то $A$-модуль $D_{n}$ изоморфен подмодулю дистрибутивного $A$-модуля $B / D$. Согласно (1) $D_{n}$ - равномерньй правый идеал.

Лемма 7. Пусть $n$ - натуральное число $и A$ - первичное кольио, являющееся суммой п дистрибутивных правых идеалов $A_{1}, \ldots, A_{n}$. Тогда имеют место следуюшие утверэддения.

(1) $A$ - конечномерное справа кольчо и правая размерность Голди кольца $A$ не превосходит числа .

(2) Если каждый правый идеал кольиа $A$ либо лежит в $J(A)$, либо содержит ненулевой идемпотент, то $A$ - полусовершенное кольцо.

(3) Если $A$ - несингулярное справа кольио, то $A$ - кольчо индекса не более $n$.

(4) Если $A$ - цорновское кольцо, то $A$ - простое артиново кольцо индекса не более $n$, изоморфное кольиу всех $(k \times k)$-матрии, над телом, где $k \leqslant n$.

ДокАЗАТЕЛьСТво. (1) следует из леммы 6(2).

(2) следует из (1) и следующего утверждения (см. $[7,4.3])$ : если $A$ - конечномерное справа кольцо и каждый правый идеал кольца $A$ либо лежит в $J(A)$, либо содержит ненулевой идемпотент, то $A$ - полусовершенное кольцо.

(3) Согласно (1) правая размерность Голди кольца $A$ не превосходит числа $n$. Кроме того, $A$ - несингулярное справа первичное кольцо. Тогда $A$ имеет простое артиново классическое правое кольцо частных $Q[1,5.10(3)]$. Кроме того, правая размерность Голди кольца $Q$ непревосходит числа $n$ и кольцо $Q$ изоморфно кольцу матрицнад телом. Поэтому $Q$ - кольцо индекса не более $n$. Тогда $A$ - кольцо индекса не более $n$.

(4) Согласно (2) $A$-полусовершенное кольцо. Последствию $1(5) A$-полупримитивное кольцо. Тогда $A$ - полупростое артиново первичное кольцо. Поэтому $A$ - простое кольцо, изоморфное кольцу всех $(k \times k)$-матриц над телом. Согласно $(3) A$ - кольцо индекса не более $n$. Поэтому $k \leqslant n$. 
ПРЕДЛОЖЕНИЕ 6. Пусть $A-\pi$-регулярное полупервичное кольцо, являющееся суммой $n$ дистрибутивных правых идеалов. Тогда $A$ - строго т-регулярное полупримитивное кольцо индекса не более $n$, каждое первичное фактор-кольио кольиа $A$ изоморфно кольиу всех $(k \times k)$-матрии над телом $(k \leqslant n)$ u $a^{n} A=a^{n+1} A$, $A a^{n}=A a^{n+1}$ для любого әлемента а кольиа $A$.

ДокАЗАтЕльство. Каждое факторкольцо $\pi$-регулярного кольца $A$ является цорновским кольцом и порождается $n$ дистрибутивными правыми идеалами. По следствию $1(5) \quad A$ полупримитивно. По лемме $7(4)$ каждое первичное факторкольцо кольца $A$ является простым артиновым кольцом индекса не более $n$ и изоморфно кольцу всех $(k \times k)$-матриц над телом, где $k \leqslant n$. Тогда полупервичное кольцо $A$ является подпрямым произведением колец индекса не более $n$. Поэтому $A$ - кольцо индекса не более $n$. Так как $A-\pi$-регулярное кольцо индекса не более $n$, то $A$ - строго $\pi$-регулярное кольцо и $a^{n} A=a^{n+1} A, A a^{n}=A a^{n+1}$ для любого элемента $a$ кольца $A$ [8].

СлЕДСТВИЕ 2. Каждое дистрибутивно порожденное справа т-регулярное кольио строго т-регулярно.

Следствие 2 вытекает из предложения 6 и того, что $A$ - строго $\pi$-регулярное кольцо $\Longleftrightarrow$ все первичные факторкольца кольца $A$ строго $\pi$-регулярны [9].

ЗАмечание $1[1,6.24(14)]$. Пусть $N$ - модуль над коммутативным кольцом $A$. Тогда $N$ - дистрибутивный $A$-модуль $\Longleftrightarrow$ для любого максимального идеала $M$ кольца $A$ модуль частных $N_{M}$ является дистрибутивньм модулем над кольцом частных $A_{M}$.

ЗАмЕчАниЕ 2. Каждое коммутативное дистрибутивно порожденное кольцо $A$ является дистрибутивным кольцом.

ДокАЗАТЕЛЬСТво. По замечанию 1 достаточно доказать, что кольцо частных $A_{M}$ дистрибутивно для любого максимального идеала $M$ кольца $A$. Пусть $A$ - сумма дистрибутивных идеалов $A_{1}, \ldots, A_{n}$ и $B_{i}$ - локализация идеала $A_{i}$ относительно $M(i=$ $1, \ldots, n)$. По замечанию 1 все $A_{M}$-модули $B_{i}$ дистрибутивны. Кроме того, $A_{M}=$ $\sum_{i=1}^{n} B_{i}$. Тогда $A_{M}$ - локальное дистрибутивно порожденное кольцо. По лемме $1(7)$ $A_{M}$ - дистрибутивное кольцо.

ЗАмечАниЕ 3 . Пусть $A$ - кольцо без единицы, $\mathbb{Z}$ - кольцо целых чисел и $A^{1}-$ прямое произведение аддитивных групп колец $A$ и $\mathbb{Z}$. Определим умножениепар $\left(a_{1}, z_{1}\right),\left(a_{2}, z_{2}\right)$ $\in A^{1}$ правилом

$$
\left(a_{1}, z_{1}\right) \cdot\left(a_{2}, z_{2}\right)=\left(a_{1} a_{2}+z_{2} a_{1}+z_{1} a_{2}, z_{1} z_{2}\right) .
$$

Группа $A^{1}$ является кольцом с единицей $(0,1)$.

Непосредственно проверяется, что кольцо $A$ изоморфно подкольцу $(A, 0)$ кольца $A^{1}$, $(A, 0)$ - идеал кольца $A^{1}$ и каждьй правьй $A$-модуль $M$ можно рассматривать как правый модуль над кольцом $A^{1}$ с единицей, если определить умножение $m \cdot(a, z)$ правилом $m \cdot(a, z)=m a+z m$.

(1) Для каждого $A$-модуля $M$ решетка всех $A$-подмодулей модуля $M$ совпадает с решеткой всех $A^{1}$-подмодулей модуля $M$. Следовательно, $M$ - дистрибутивньй $A$-модуль $\Longleftrightarrow M-$ дистрибутивньй $A^{1}$-модуль.

(2) Для любых двух правых $A$-модулей $M$ и $N$ множество всех $A$-гомоморфизмов $M \rightarrow N$ совпадает с множеством всех $A^{1}$-гомоморфизмов $M \rightarrow N$. Следовательно, $\operatorname{End}\left(M_{A}\right)=\operatorname{End}\left(M_{A^{1}}\right)$. 


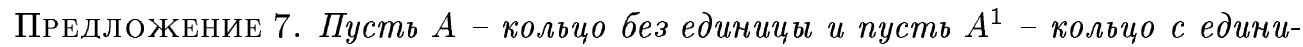
цей, построенное в замечании 3. Тогда имеют место следуюшие утверждения.

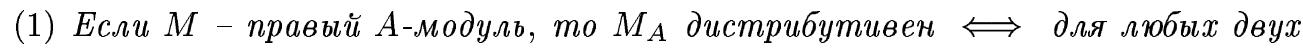
әлементов $m_{1}, m_{2} \in M$ существуют иелые числа $z, y_{1}, y_{2}$ и элементы $a, b_{1}, b_{2} \in A$ такие, что $m_{1} a+z m_{1}=m_{2} b_{2}+y_{2} m_{2}, m_{2}-m_{2} a-z m_{2}=m_{1} b_{1}+y_{1} m_{1}$.

(2) $A^{1}$ - дистрибутивное справа кольцо $\Longleftrightarrow A^{1}-$ дистрибутивный правый $A$-модуль $\Longleftrightarrow$ для любых әлементов $a_{1}, a_{2} \in A$ и любых иелых чисел $x_{1}, x_{2}$ существуют иелье числа $z, x_{1}, x_{2}, y_{1}, y_{2}$ и әлементы $a, b_{1}, b_{2} \in A$ такие, что $a_{1} a+x_{1} a+z a_{1}=a_{2} b_{2}+x_{2} b_{2}+y_{2} a_{2}, a_{2}-a_{2} a-x_{2} a-z a_{2}=a_{1} b_{1}+x_{1} b_{1}+y_{1} a_{1}$, $z x_{1}=y_{2} x_{2}, x_{2}-z x_{2}=y_{1} x_{1}$.

(3) Если $M$ - дистрибутивный $A$-модуль, то $\operatorname{End}(M)$ - нормальное кольцо, $y$ которого все односторонние ниль-идеалы лехсат в первичном радикале.

(4) Если $B$ - дистрибутивный правый идеал кольца $A$ и $X, Y$ - правые идеаль кольиа $A$ такие, что $X \subseteq B, Y \subseteq B$ и $X \cap Y=0$, mо $X A Y+Y A X+X Y=0$.

(5) Если $B$ - дистрибутивный правый идеал кольца $A$ и $M$ - максимальный подмодуль модуля $B_{A}$, то $M-$-идеал кольиа $B$ без единицы.

ДокАЗАТЕЛьСТво. (1) следует из замечания 3(1) и леммы 5(1).

(2) следует из (1) и замечания $3(1)$.

(3) следует из леммы $5(2)$ и замечания 3.

(4) следует из замечания $3(1)$ и того, что $\left(X A^{1}\right)\left(Y A^{1}\right)=0$ в силу $[1,1.50(1)]$.

$(5)$ Для каждого элемента $b \in B$ существует эндоморфизм $f_{b} \in \operatorname{End}\left(B_{A}\right)$ такой, что $f_{b}(x)=b x$ для всех $x \in B$. По лемме $5(2)$ и замечанию $3 M-$ вполне инвариантный подмодуль модуля $B_{A}$. Поэтому $B M \subseteq M$ и $M$ - идеал кольца $B$.

\section{СПИСОК ЦИТИРОВАННОЙ ЛИТЕРАТУРЫ}

[1] Tuganbaev A. A. Semidistributive Rings and Modules. Dordrecht-Boston-London: Kluwer Acad. Publ., 1998.

[2] Mazurek R., Puczylowski E. R. On semidistributive rings // Comm. Algebra. 1997. V. 25. № 11. P. 3463-3471.

[3] Warfield R. B. Exchange rings and decompositions of modules // Math. Ann. 1972. V. 199. P. 31-36.

[4] Dischinger F. Sur les anneaux fortement $\pi$-réguliers // C. R. Acad. Sci. 1976. V. 283. № 8. P. A571-A573.

[5] Кириченко В.В.О полуцепных наследственных и полунаследственных кольцах // Записки научных семинаров ЛОМИ. 1982. Т. 114. С. 137-146.

[6] Фейс К. Алгебра: кольца, модули и категории 1. М.: Мир, 1977.

[7] Nicholson W. K. I-rings // Trans. Amer. Math. Soc. 1975. V. 207. P. 361-373.

[8] Azumaya G. Strongly $\pi$-regular rings // J. Fac. Sci. Hokkaido Univ. 1954. V. 13. P. 659-672.

[9] Fisher J.W., Snider R.L. On the Von Neumann regularity of rings with regular prime factor rings // Pacific J. Math. 1974. V. 54. №1. P. 135-144.

Московский энергетический институт

Поступило

E-mail : askar@tuganbaev.mccme.ru

24.12.1998

Исправленный вариант

15.12.1999 